\title{
Coding for Frequency-Hopped Spread-Spectrum Communication with Partial-Band Interference-Part I: Capacity and Cutoff Rate
}

\author{
WAYNE E. STARK, MEMBER, IEEE
}

\begin{abstract}
The performance of optimal codes on frequency-hopped channels with partial-band interference is investigated. The performance measures considered are channel capacity and cutoff rate. Worst-case partial-band Gaussian noise interference is assumed with the interference independent of the transmitted signal. The capacity and cutoff rate are calculated as a function of the signal-to-noise ratio. We consider soft decision receivers and hard decision receivers with and without side information. Optimal code rates are found for each of the above cases. The required signal-to-noise ratio for reliable communication when codes are used is determined as a function of the code rate.
\end{abstract}

\section{INTRODUCTION}

$\mathrm{O}$ VER the last several years there has been considerable interest in the problem of coding for channels with partial-band interference. Many authors have considered the performance of codes on such channels [1]-[4]. Some work has also been done in computing the computational cutoff rate for certain of these channels [5]-[9]. These papers show that the use of coding is extremely important when considering the worst case performance against an intelligent jammer. Evaluation of the coded error probabilities [7], [10], [11] for antijam communication systems show that gains on the order of $30-40 \mathrm{~dB}$ can be obtained over uncoded systems.

There are several key issues that arise when considering coding for spread-spectrum communications in the presence of partial-band interference. One issue is whether or not the decoder knows if the received signal has been jammed or not. Naturally, the decoder knowing and using this side information in a clever way can improve the performance compared to coding without side information available. Another issue that must be addressed is that of interleaving. If we are considering a fast frequency-hopped (FFH) spread-spectrum (SS) communication system, then there is just one symbol transmitted per hop and interleaving is unnecessary. However, for slow frequency-hopped (SFH) spread-spectrum communication with multiple symbols per hop, the symbols in a single hop are subject to the same type of interference, and thus, the errors that occur on different symbols in the same hop are dependent. Interleaving these symbols breaks up the memory of the channel and allows the use of randomerror correcting codes. Alternatively, burst-error correcting codes could be used without interleaving. The papers that consider error-correction usually consider only random-error correcting codes with interleaving (or fast frequency hopping).

In this paper we examine the performance of codes in

Paper approved by the Editor for Communication Systems Disciplines of the IEEE Communications Society. Manuscript received June 2, 1983; revised November 23, 1984. This paper was presented in part at the National Telecommunications Conference, New Orleans, LA, December 1981. This work was supported in part by the Joint Services Electronics Program under Grant N00014-79-C-0424.

The author is with the Department of Electrical Engineering and Computer Science, University of Michigan, Ann Arbor, Ml 48109. terms of the channel capacity and channel cutoff rate. We consider the following cases.

- SFH (with one or more symbols per hop)

Side information available

- No side information available

\section{Soft decisions}

- Hard decisions.

We model the interference (jammer) as adding noise to the transmitted signal. We also make the assumption that the jamming signal is independent of the transmitted signal (i.e., repeat-back jammers and frequency follower jammers are not considered). In Section II we describe the channel models that will be used in the analysis. In Section III the capacity of SFH channels will be derived. In Section IV the corresponding analysis will be made when the cutoff rate is used as the performance criterion. Finally, in Section $V$ we will give numerical results and draw conclusions. In Part II of this paper the performance of specific codes will be analyzed.

\section{Channel Models}

The block diagram of the communication system we are considering is shown in Fig. 1. The model we employ for the frequency hopper and dehopper is that of [12] and will be summarized here. The data signal $b(t)$ is a sequence of nonoverlapping rectangular pulses of duration $T$. The amplitude of the $l$ th pulse of $b(t), l T \leq t<(l+1) T$, is the random variable $X_{l}$ which takes values in the input alphabet $A$. For MFSK with $M$ orthogonal tones, $A=\{0,1, \cdots, M$ $-1\}$. We treat the sequence $X_{i}, i \geq 0$, as the coding channel input (shown in dotted lines in Fig. 1).

As in [12] the MFSK modulator output, when $b(t)$ is the input, is the signal

$$
c(t)=\cos \left(2 \pi\left[f_{c}+b(t) \Delta\right] t+\theta(t)\right)
$$

where $\Delta$ is the spacing between MFSK tones and $\theta(t)$ is the phase signal introduced by the modulator. If $b(t)=j, l T \leq t$ $<(l+1) T$, then $\theta(t)=\theta_{j}$ for $l T \leq t<(l+1) T$, where $\theta_{j}$ is a random phase. We note here that, while we described the modulator for MFSK as that of $M$ orthogonal tones, the results presented here are applicable to any set of $M$ orthogonal signals.

The frequency hopper then changes the frequency according to a hopping pattern $f(t)$ which is a sequence of nonoverlapping rectangular pulses of duration $T_{h}$ with $f(t)=$ $f_{j}, j T_{h} \leq t \leq(j+1) T_{h}$, where $T_{h}$ is the time between hops and $f_{j} \in S=\left\{\nu_{1}, \nu_{2}, \cdots, \nu_{q}\right\}$. The set $S$ is the set of frequencies to which the signal is allowed to hop. Some necessary restrictions on $S$ are given in [12] for MFSK with $M=2$, which may be easily extended for $M>2$. The number of symbols per hop is taken to be $N_{s}$ where $N_{s}=$ 
CODING CHANNEL

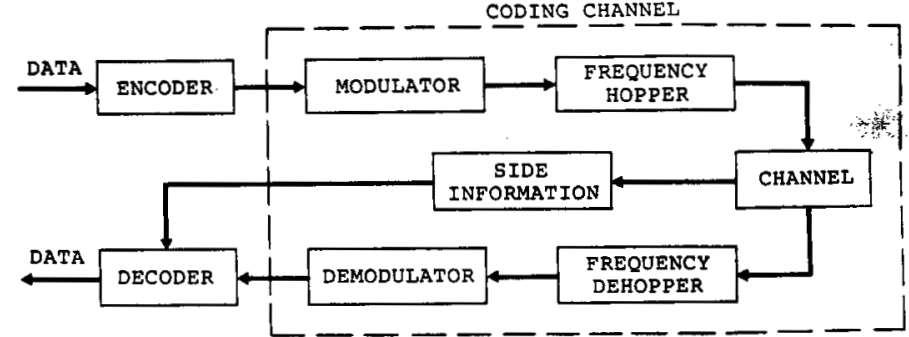

Fig. 1. Communication system block diagram.

$T_{h} / T$. The output of the frequency hopper is the waveform

$$
s(t)=\sqrt{2 P} \cos (2 \pi \hat{f}(t) t+\phi(t))
$$

where

$$
\hat{f}(t)=f_{c}+b(t) \Delta+f(t)
$$

and

$$
\phi(t)=\theta(t)+\alpha(t)
$$

In (4) $\alpha(t)$ is the random phase signal introduced by the frequency hopper with $\alpha(t)=\alpha_{j}$ in the interval $j T_{h} \leq t \leq(j$ $+1) T_{h}$. In (2) $P$ is the power of the received signal. See [12] for further discussion of the signal models used.

The jamming signal $j(t)$ at the receiver will be modeled as a weighted sum of bandpass Gaussian processes:

$$
j(t)=\sum_{i=1}^{q} Z_{i}(t) j_{i}(t)
$$

where $\left\{j_{i}(t): 0 \leq t \leq \infty\right\}$ is a Gaussian noise process with spectral density $N_{J}$ over a bandwidth of $W / q \mathrm{~Hz}$ centered at frequency $f_{c}+\nu_{i}+(M-1) \Delta / 2$. Here $W$ is the total spread bandwidth of the transmitted signal. The power of each signal, $j_{i}(t)$, is then $N_{J} W / q$. In (5) $Z_{i}(t)$ is a sequence of nonoverlapping rectangular pulses of duration $n T_{h}$, where $Z_{i}(t)=Z_{i, l}$ for $l T_{h} \leq t<(l+1) T_{h}$. We assume that the spectral density $S_{i}(\omega)$ of $j_{i}(t)$ is such that $S_{i}(\omega) S_{k}(\omega)=0$ for all $\omega$ and $i \neq k$. Thus, $j_{i}(t)$ and $j_{k}(t)$ are independent random processes for $i \neq k$.

The jammer has the freedom to choose the distribution of the random variables $Z_{i, l}$ subject to an average power constraint:

$$
E\left[\sum_{i=1}^{q} Z_{i, l^{2}}\right] \leq q
$$

The strategy for a partial band jammer is to choose the following distribution for $Z_{i, l}$ :

$$
\begin{array}{ll}
P\left\{Z_{i, l}=0\right\}=1-\rho & 0<\rho \leq 1 \\
P\left\{Z_{i, l}=\sqrt{1 / \rho}\right\}=\rho &
\end{array}
$$

where $\rho$ is a constant that represents the fraction of band that has interference. The average power constraint (6) with the assumption that $Z_{i}(t)$ and $j_{i}(t)$ are independent yields total average power of the interference of $N_{J} W$. Also, for each $i$, $Z_{i, l}$ is a sequence of independent, identically distributed random variables with statistics given by (7). The received signal $r(t)$ is then given by

$$
r(t)=s(t)+j(t)+n(t)
$$

where $n(t)$ is the thermal noise, which is a white Gaussian noise process with spectral density $N_{0} / 2$. The received signal is first dehopped by an ideal frequency dehopper. The output of the frequency dehopper, $r_{d}(t)$, can be written as

$$
\begin{aligned}
& r_{d}(t)=\sqrt{2 P} \cos \left[2 \pi\left(f_{c}+b(t) \Delta\right) t+\phi(t)+\beta(t)\right] \\
&+\sum_{k=1}^{q} \delta\left(\nu_{k}, f(t)\right) Z_{k}(t) j_{k}(t)+n(t)
\end{aligned}
$$

In (9), $\delta(u, r)=0$ if $u \neq r$ and $\delta(u, u)=1$. Also, $n(t)$ is a band-limited white Gaussian noise process with bandwidth much larger than that of the demodulator and $\beta(t)$ is the phase signal introduced by the dehopper. The factor $\delta\left(\nu_{i}, f(t)\right)$ in the middle term of (9) represents the filtering done by the dehopper.

The demodulator we consider processes the received signal by computing the $M$-dimensional vector $Y_{j}=\left(Y_{0, j}\right.$, $\left.Y_{1, j}, \cdots, Y_{M-1, j}\right)$ where

$$
\begin{gathered}
Y_{i, j}=Y_{i, j, c^{2}}+Y_{i, j, s}{ }^{2} \\
Y_{i, j, c}=\int_{j r}^{(j+1) \tau} r_{d}(t) \cos 2 \pi\left(f_{c}+i \Delta\right) t d t
\end{gathered}
$$

and

$$
Y_{i, j, s}=\int_{j \tau}^{(j+1) r} r_{d}(t) \sin 2 \pi\left(f_{c}+i \Delta\right) t d t .
$$

For slow frequency hopping (SFH), $\tau$ in (11) is just $T$ and $N_{s}=T_{h} / T$ is the number of symbols per hop. Define the integers $l$ and $n$ by $0 \leq l<N_{s}$ and $j=n N_{s}+l$. Then $Y_{j}$ is the channel output of the $l$ th symbol of the $n$th hop. Then, using (9) in (11) we can write

$$
\begin{aligned}
Y_{i, j, c}=\sqrt{P / 2} \delta\left(X_{j},\right. & i) T \cos \left(\Psi_{j}\right) \\
& +\sum_{k=1}^{q} \delta\left(\nu_{k}, f_{n}\right) Z_{k, n} \eta_{k, j, s}+\hat{\eta}_{j, s} \\
Y_{i, j, s}=\sqrt{P / 2} \delta\left(X_{j},\right. & i) T \cos \left(\Psi_{j}\right) \\
& +\sum_{k=1}^{q} \delta\left(\nu_{k}, f_{n}\right) Z_{k, n} \eta_{k, j, s}+\hat{\eta}_{j, s}
\end{aligned}
$$

where

$$
\Psi_{j}=\theta_{j}+\alpha_{n}+\beta_{n}
$$

Also in (12) $\eta_{j, k, c}, \eta_{j, k, s}, \hat{\eta}_{j, c}$, and $\hat{\eta}_{j, s}$ are independent sequences of independent, identically distributed (i.i.d.) zero-mean Gaussian random variables. The variance of $\eta_{k, j, c}$ and $\eta_{k, j, s}$ is $N_{J} T / 4$ while the variance of $\hat{\eta}_{j, c}$ and $\hat{\eta}_{j, s}$ is $N_{0} T / 4$. Notice in (12) that the random variables subscripted with $n$ change only every hop, whereas those subscripted with $j$ change on every symbol of every hop. The random variables that are fixed for an entire hop cause the channel to have memory. However, conditioned on these random variables, the channel is memoryless. The channel is memoryless from hop to hop and fits exactly the model in [13] of channels with memory. Let $S_{n}$ be a sequence of random variables defined as follows. If $f_{n}=\nu_{k}$ for some $k, 1 \leq k \leq q$, then $S_{n}=1$ if $Z_{k, n} \neq 0$ and $S_{n}=0$ if $Z_{k, n}=0$. The distribution of $S_{n}$ is then

$$
\begin{gathered}
P\left\{S_{n}=0\right\}=1-\rho \\
P\left\{S_{n}=1\right\}=\rho .
\end{gathered}
$$


The random variables in (12) can easily be shown to be Gaussian when conditioned $X_{j}, \Psi_{j}$, and $S_{n}$ with mean

$$
\begin{aligned}
& E\left[Y_{i, j, c} \mid X_{j}, \Psi_{j}, S_{n}\right]=\sqrt{P / 2} \delta\left(X_{j}, i\right) T \cos \Psi_{j} \\
& E\left[Y_{i, j, s} \mid X_{j}, \Psi_{j}, S_{n}\right]=\sqrt{P / 2} \delta\left(X_{j}, i\right) T \sin \Psi_{j}
\end{aligned}
$$

and variance

$$
\begin{array}{r}
\operatorname{var}\left(Y_{i, j, c} \mid X_{j}, \Psi_{j}, S_{n}\right)=\frac{1}{4} N_{J} T S_{n} / \rho+\frac{1}{4} N_{0} T \\
\operatorname{var}\left(Y_{i, j, s} \mid X_{j}, \Psi_{j}, S_{n}\right)=\frac{1}{4} N_{J} T S_{n} / \rho+\frac{1}{4} N_{0} T .
\end{array}
$$

The conditional density of $Y_{i, j}$ can be computed as

$$
\begin{aligned}
p\left(y_{i j} \mid X_{j}=l, S_{n}\right) & \begin{cases}\frac{1}{2 \sigma^{2}} \exp \left[-\left(\frac{y_{i j}+\Lambda}{2 \sigma^{2}}\right)\right] I_{0}\left(\sqrt{y_{i j} \Lambda} / \sigma^{2}\right) & l=i \\
\frac{1}{2 \sigma^{2}} \exp \left[-\frac{y_{i j}}{2 \sigma^{2}}\right] & l \neq i\end{cases}
\end{aligned}
$$

where

$$
\begin{gathered}
\sigma^{2}=\frac{1}{4} S_{n} N_{J} T / \rho+\frac{1}{4} N_{0} T \\
\Lambda=P T^{2} / 2
\end{gathered}
$$

and $I_{0}(x)$ is the modified Bessel function of order 0 .

The joint density of the random vector $Y_{j}$ when conditioned on $X_{j}$ and $S_{n}$ is given by

$$
p\left(y_{j} \mid X_{j}=l, S_{n}\right)=\prod_{i=0}^{M-1} p\left(y_{i j} \mid X_{j}=l, S_{n}\right) .
$$

Furthermore, the channel with input $X_{j}$ and output $Y_{j}$ is a memoryless channel only when conditioned on $S_{n}$. However, if we let $\boldsymbol{X}^{(n)}=\left(\boldsymbol{X}_{n N_{s}}, \boldsymbol{X}_{n N_{s}+1}, \cdots, \boldsymbol{X}_{(n+1) N_{s}-1}\right)$ and $\boldsymbol{Y}^{(n)}=$ $\left(\boldsymbol{Y}_{n N_{s}}, \boldsymbol{Y}_{n N_{s}+1}, \cdots, \boldsymbol{Y}_{\left((n+1) N_{s}-1\right)}\right)$, then the channel with input $X^{(n)}$ and output $Y^{(n)}$ is a memoryless channel.

The channel with input $X^{(n)}$ and output $Y^{(n)}$ is called the channel with soft decisions and no side information. If in addition to $Y^{(n)}, S_{n}$ is an output of the channel, then this is called the channel with soft decisions and side information. If the receiver after computing $Y_{j}$ chooses the index of the largest component as the transmitted symbol, then the receiver has made a hard decision. The probability that the receiver makes an error on any symbol of the $n$th hop is given by

$P_{e, s}^{(n)}=\frac{1}{M} \sum_{j=2}^{M}(-1)^{j}\left(\begin{array}{c}M \\ j\end{array}\right) \exp \left\{-\frac{E}{\left(S_{n} N_{J} / \rho+N_{0}\right)}(1-1 / j)\right\}$

where $E=P T$. For hard decisions without side information available, the channel output is just the index of the largest component of $Y_{j}$ which, for each $j=n N_{s}+l$, is just an $M$ ary symmetric channel with transition probability $\boldsymbol{P}_{e, s}{ }^{(n)}$. For hard decisions with side information available, the channel output is the index of the largest component of $Y_{j}$ and the value of $S_{n}$.

For the purposes of this part of the paper, we will assume that the thermal noise is negligible (i.e., $N_{0}=0$ ). However, in Part II of the paper this will not be assumed. When $N_{0}=0$ and $S_{n}=0,(17)$ is no longer valid. In this case $Y_{i j}=\left(P T^{2} /\right.$ 2) $\delta\left(X_{j}, i\right)$. For $N_{0}=0$ and $S_{n} \neq 0, P\left\{Y_{i j}=\left(P T^{2} / 2\right) \delta\left(X_{j}\right.\right.$, $i)\}=0$ so that side information is available implicitly. For other decoding rules (suboptimal decoding), soft decisions may not imply that side information is present. For these soft decision decoding rules with no side information, the performance is generally poor when there is a partial band jammer (see [14]).

\section{SFH CHANNEL CAPACITY}

In this section we compute the capacity of a slow frequency-hopped spread-spectrum system subject to partialband jamming. We first consider the case of side information present, then the case of no side information present. When side information is present, the channel capacity is independent of the number of bits per hop or the channel memory [13]. Without side information the channel capacity is less than the capacity with side information, and increases to the capacity with side information as $N_{s}$ becomes large [13].

The capacity of the channel is obtained from a game theoretic formulation in which the transmitter (coder) is allowed to choose any distribution on the channel input, and the jammer is allowed to choose any $\rho \in(0,1]$ as the fraction of the band that is jammed. The payoff function of the game is the mutual information between the channel input and output. The coder chooses his distribution on $X^{(n)}$ in order to maximize the mutual information, while the jammer chooses $\rho \in(0,1]$ in order to minimize the mutual information. The channel capacity is then given by

$$
C=\max _{X^{(n)}} \min _{\rho} I\left(X^{(n)} ; \quad Y^{(n)}\right)=\min _{\rho} \max _{X^{(n)}} I\left(X^{(n)} ; Y^{(n)}\right)
$$

when no side information is available, and when side information is present the capacity is given by

$$
\bar{C}=\max _{X^{(n)}} \min _{\rho} I\left(X^{(n)} ; Y^{(n)}, S_{n}\right)=\min _{\rho} \max _{X^{(n)}} I\left(X^{(n)} ; Y^{(n)}, S_{n}\right) .
$$

In [15] it has been shown that the optimal distribution on $X^{(n)}$ or coding strategy is to choose $X^{(n)}$ to be a vector of i.i.d. random variables. It also can be shown $[16, p .64]$ that the optimal distribution of $X_{j}$ is the uniform distribution on $A$, since this is a symmetric channel. Thus, we let $X_{j}$ have uniform distribution on $A$. The justification of $C$ and $\bar{C}$ as the channel capacity is given in the Appendix.

\section{A. Side Information Available}

First consider the case of side information available. In this case the capacity is independent of the number of bits per hop, so we assume without loss of generality that $N_{s}=1$. With this assumption the mutual information is given by

$$
\begin{aligned}
I\left(X_{j} ; Y_{j}, S_{n}\right)=\rho I\left(X_{j} ; Y_{j} \mid\right. & \left.S_{n}=1\right) \\
& +(1-\rho) I\left(X_{j} ; Y_{j} \mid S_{n}=0\right) .
\end{aligned}
$$

Furthermore, if $S_{n}=0$ then there is no noise so that $I\left(X_{j}\right.$; $\left.Y_{j} \mid S_{n}=0\right)=1$ symbol when the coder chooses the uniform distribution on $A$. When $S_{n}=1$ the mutual information is determined from the channel statistics given in (17) and (18):

$$
\begin{aligned}
I\left(X_{j} ; Y_{j} \mid S_{n}=1\right)=\int_{y_{j}} p\left(y_{j} \mid X_{j}=x, S_{n}=1\right) \\
\cdot \log _{M} \frac{p\left(y_{j} \mid X_{j}=x, S_{n}=1\right)}{p\left(y_{j} \mid S_{n}=1\right)} d y_{j} .
\end{aligned}
$$


The above integral is an $M$-dimensional integral which can be evaluated numerically for $M=2$. For $M>2$ numerical evaluation becomes computationally difficult. To compute the capacity, however, requires the computation of $\min I\left(X_{j}\right.$; $\left.\boldsymbol{Y}_{j}, S_{n}\right)$ :

$$
\min _{\rho} I\left(X_{j} ; Y_{j}, S_{n}\right)=\min _{\rho}\left[(1-\rho)+\rho C_{M}\left(E \rho / N_{J}\right)\right]
$$

where

$$
C_{M}\left(E \rho / N_{J}\right)=I\left(X_{j} ; Y_{j} \mid S_{n}=1\right)
$$

Here we take as our unit of capacity $M$-ary information symbols/ $M$-ary channel symbol. For the minimum of (23), the worst case $\rho, \rho^{*}$, satisfies

$$
\rho^{*}=\left\{\begin{array}{cl}
1, & E / N_{J}<\bar{\Upsilon}_{M} \\
\frac{\bar{\Upsilon}_{M}}{E / N_{J}}, & E / N_{J} \geq \bar{\Upsilon}_{M}
\end{array}\right.
$$

where $\bar{\Upsilon}_{M}$ is the solution to

$$
C_{M}\left(\bar{\Upsilon}_{M}\right)+\bar{\Upsilon}_{M} C_{M}^{\prime}\left(\bar{\Upsilon}_{M}\right)=1
$$

With $\rho$ given in (25), the minimum mutual information is given by

$$
\min _{\rho} I\left(X_{j} ; Y_{j}, S_{n}\right)=\left\{\begin{array}{cl}
C_{M}\left(E / N_{J}\right), & E / N_{J}<\bar{\Upsilon}_{M} \\
1-\frac{\bar{\Upsilon}_{M}}{E / N_{J}}\left[1-C_{M}\left(\bar{\Upsilon}_{M}\right)\right], & E / N_{J}>\bar{\Upsilon}_{M}
\end{array}\right.
$$

Since the capacity is the maximum of the minimum mutual information and is achieved by a uniform input distribution, we have

$$
\bar{C}\left(E / N_{J}\right)=\left\{\begin{array}{cc}
C_{M}\left(E / N_{J}\right), & E / N_{J}<\bar{\Upsilon}_{M} \\
1-\frac{\bar{\Upsilon}_{M}\left[1-C_{M}\left(\bar{\Upsilon}_{M}\right)\right]}{E / N_{J}}, & E / N_{J}>\bar{\Upsilon}_{M}
\end{array}\right.
$$

For binary FSK with soft decisions, $C_{2}(x)$ has been computed as [17]

$$
\begin{aligned}
C_{2}(x)=e^{-x^{2}} & \int_{0} \int_{0}^{\infty} y_{0} y_{1} \\
\cdot & \exp \left\{-\left(y_{0}^{2}+y_{1}^{2}\right)\right\} f\left(x, y_{0}, y_{1}\right) d y_{0} d y_{1}
\end{aligned}
$$

where

$$
f\left(x, y_{0}, y_{1}\right)=I_{0}\left(2 x y_{0}\right) \log _{2}\left\{\frac{2 I_{0}\left(2 x y_{0}\right)}{I_{0}\left(2 x y_{0}\right)+I_{0}\left(2 x y_{1}\right)}\right\} .
$$

Using (29) in (28) we have, for soft decisions with side information,

$$
\bar{C}_{2}\left(E / N_{J}\right)= \begin{cases}C_{2}\left(E / N_{J}\right) & E / N_{J}<2.4137 \\ 1-\frac{1.1741}{E / N_{J}} & E / N_{J} \geq 2.4137\end{cases}
$$

When hard decisions are made, the channel with $S_{n}=1$ is just an $M$-ary symmetric channel with crossover probability given by

$P_{e, s}\left(E \rho / N_{J}\right)=\frac{1}{M} \sum_{j=2}^{M}(-1)^{j}\left(\begin{array}{c}M \\ j\end{array}\right) \exp \left\{-\frac{E \rho}{N_{J}}(1-1 / j)\right\}$

which is (19) with $\dot{N}_{0}=0$. The capacity $\hat{C}_{M}(p)$ of an $M$-ary symmetric channel with crossover probability $p$ is given by

$$
\hat{C}_{M}(p)=1+(1-p) \log _{M}(1-p)+p \log _{M}(p /(M-1)) .
$$

Thus, with side information available and hard decisions, the capacity of an $M$-ary FSK channel subject to partial-band jamming is given by

$$
C_{M}\left(E / N_{J}\right)=\left\{\begin{array}{cc}
\hat{C}_{M}\left(P_{e, s}\left(E / N_{J}\right)\right), & E / N_{J}<\Upsilon_{M} \\
1-\frac{\Upsilon_{M}-\Upsilon_{M} \hat{C}_{M}\left(P_{e, s}\left(\Upsilon_{M}\right)\right)}{E / N_{J}}, & E / N_{J} \geq \Upsilon_{M}
\end{array}\right.
$$

where $\Upsilon_{M}$ is a constant.

\section{B. No Side Information}

Next consider the case of no side information available and hard decisions. Consider the case $N_{s}=1$. Again, the jammer's strategy is to choose $\rho$ to minimize the mutual information. However, because the capacity is a decreasing function of the error probability when the error probability is less than $(M-1) / M$, an equivalent strategy for the jammer is to maximize the error probability. The error probability is given by the average of the error probability when $S_{n}=1$ and the error probability when $S_{n}=0$.

$$
\bar{P}_{e, s}\left(E / N_{J}, \rho\right)=\rho P_{e, s}\left(E \rho / N_{J}\right)+(1-\rho) 0 \text {. }
$$

The error probability is assumed to be 0 when $S_{n}=0$. The maximum error probability is given by

$$
\bar{P}_{e, s}\left(E \rho / N_{J}\right)=\max _{0<\rho \leq 1} \rho P_{e, s}\left(E \rho / N_{J}\right)
$$

$$
\bar{P}_{e, s}\left(E / N_{J}\right)= \begin{cases}P_{e, s}\left(E / N_{J}\right) & E / N_{J}<\lambda_{M} \\ \frac{\lambda_{M} P_{e, s}\left(\lambda_{M}\right)}{E / N_{J}} & E / N_{J} \geq \lambda_{M}\end{cases}
$$

where $\lambda_{M}$ is a constant. This constant $\lambda_{M}$ is the same as that found by Houston [1] when computing the worst case partialband jammer for $M$-ary FSK. The capacity is then found using (32) as

$$
C_{M}\left(E / N_{J}\right)=\left\{\begin{array}{lr}
\hat{C}_{M}\left(P_{e, s}\left(E / N_{J}\right)\right) & E / N_{J}<\lambda_{M} \\
\hat{C}_{M}\left(\frac{\lambda_{M} P_{e, s}\left(\lambda_{M}\right)}{E / N_{J}}\right) & E / N_{J} \geq \lambda_{M}
\end{array}\right.
$$

When $N_{s}>1$, the channel exhibits memory since all bits during a particular hop are either jammed or not jammed. If no side information is available, then the channel capacity depends on the memory length $N_{s}$. When the receiver makes hard decisions, the capacity for $M$-ary FSK is calculated as 
[15, Appendix A]

$$
\begin{aligned}
C_{M}\left(N_{s}\right)=\min _{0<\rho \leq 1} & \left\{1+\frac{1}{N_{s}} \sum_{k=0}^{N_{s}}\right. \\
& \left.\cdot\left(\begin{array}{c}
N_{s} \\
k
\end{array}\right)(M-1)^{k} \alpha_{N_{s}, k} \log _{M} \alpha_{N_{s}, k}\right\}
\end{aligned}
$$

where $\alpha_{m, k}$ is defined as

$\alpha_{m, k}= \begin{cases}(1-\rho)+\rho\left(1-P_{e, s}\left(E \rho / N_{J}\right)\right)^{m}, \quad k=0 & \\ \rho\left(1-P_{e, s}\left(E \rho / N_{J}\right)\right)^{m-k} P_{e, s}{ }^{k}\left(E \rho / N_{J}\right), & k \neq 0 .\end{cases}$

The minimization in (37) must be done numerically for $N_{s}>$ 1 .

\section{SFH Channel Cutoff Rate}

In this section we derive the computational cutoff rate for channels with partial-band jamming and noncoherent demodulation. We consider both cases of side information available. Some of these results have appeared previously in the literature [6], [7], [9] for memoryless channels $\left(N_{s}=1\right)$. Here we present new results on the cutoff rate for channels with memory (i.e., $N_{s}>1$ ). In [7] and [9] a different definition (based on suboptimal decoding) of cutoff rate is used for the case of soft decisions than that used here. We consider the cutoff rate based on log-likelihood decoding and compare the result to that in [7] and [9]. We find that for soft decisions the difference is $0.14 \mathrm{~dB}$ in signal-to-noise ratio. We derive the form of the cutoff rate when evaluated for the worst case partial-band interference and determine the optimal code rate for minimizing the signal-to-noise ratio required for reliable performance. The cutoff rate is considered for three cases: i) side information available, soft decisions, ii) side information available, hard decisions, and iii) no side information available, hard decisions. For cases ii) and iii) the cutoff rate is also determined when the channel has memory.

\section{A. Side Information A vailable}

We consider first the case of side information available. Unlike the channel capacity, the cutoff rate with side information depends on the memory of the channel. From [13] the cutoff rate with side information can be written as

$$
\bar{R}_{0}\left(N_{s}\right)=\min _{0<p \leq 1}\left\{-\frac{1}{N_{s}} \log _{M} E\left[J^{N_{s}}\left(S_{n}\right)\right]\right\}
$$

where

$$
J(s)=M^{-R_{0, s}}
$$

and $R_{0, s}$ is the cutoff rate of the channel when $S_{n}=s$. In deriving (39) and (40) it was assumed that the input distribution that achieves the cutoff rate is the same for both channels. This is valid for all cases considered here.

When $S_{n}=1$ the channel statistics are that of an $M$-ary FSK additive Gaussian noise channel [see (17)] with signalto-noise ratio $E \rho / N_{J}$. The cutoff rate $R_{0}\left(E \rho / N_{J}\right)=$ $-\log _{M} J_{M}\left(E \rho / N_{J}\right)$ is achieved with a uniform input distribution on $A$. When the jammer is off, the channel is noiseless, so the cutoff rate is 1 (measured in $M$-ary units) and is also achieved by a uniform input distribution. The cutoff rate of the composite channel (with $N_{s}=m$ ), measured in $M$-ary information symbols/ $M$-ary channel symbol, is then

$$
R_{0, M^{(m)}}\left(E / N_{J}\right)=-\frac{1}{m} \log _{M} \bar{J}_{M}^{(m)}\left(E / N_{J}\right)
$$

where

$$
\bar{J}_{M}^{(m)}\left(E / N_{J}\right)=\max _{0<\rho \leq 1} \rho J_{M}^{m}\left(E \rho / N_{J}\right)+(1-\rho)\left(\frac{1}{M}\right)^{m} .
$$

The worst case value of $\rho=\rho^{*}$ has the form

$$
\rho^{*}=\left\{\begin{array}{cl}
1, & E / N_{J}<\sigma_{M, m} \\
\frac{\sigma_{M, m}}{E / N_{J}}, & E / N_{J} \geq \sigma_{M, m}
\end{array}\right.
$$

where $\sigma_{M, m}$ is the solution of

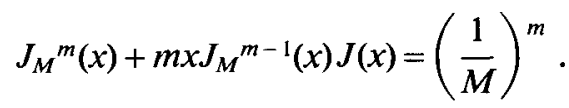

When (43) is used in (41), we obtain

$$
\bar{J}_{M}^{(m)}\left(E / N_{J}\right)= \begin{cases}J_{M}\left(E / N_{J}\right), & E / N_{J}<\sigma_{M, m} \\ \left(\frac{1}{M}\right)^{m}+\frac{\Sigma_{M, m}}{E / N_{J}}, E / N_{J}>\sigma_{M, m}\end{cases}
$$

where $\Sigma_{M, m}=\sigma_{M, m} J_{M}^{m}\left(\sigma_{M, m}\right)-M^{-m} \sigma_{M, m}$. From (45) and (41) we obtain $R_{0, M}^{(m)}\left(E / N_{J}\right)$ as

$$
R_{0, M}^{(m)}\left(E / N_{J}\right)=\left\{\begin{array}{c}
R_{0, M}\left(E / N_{J}\right), \quad E / N_{J}<\sigma_{M, m} \\
1-\frac{1}{m} \log _{M}\left(1+\frac{M^{m} \Sigma_{M, m}}{E / N_{J}}\right), \\
E / N_{J} \geq \sigma_{M, m} .
\end{array}\right.
$$

The constants $\Sigma_{M, m}$ and $\sigma_{M, m}$ depend on the type of receiver (e.g., hard decisions or soft decisions) and the memory length of the channel.

When the receiver does not do any quantization (i.e., soft decisions), then $J_{M}(x)$ for an additive white Gaussian noise channel with $E / N_{J}=x$ is given by [18]

$$
J_{M}(x)=\frac{1+(M-1) e^{-x}\left[\int_{0}^{\infty} t e^{-t^{2} / 2} I_{0}^{1 / 2}(t \sqrt{2 x}) d t\right]^{2}}{M}
$$

For $m=1$ it is easy to check that the maximization in (42) is independent of $M$. In this case $(m=1)$ the cutoff rate is given by

$$
\bar{R}_{0, M}^{(1)}\left(E / N_{J}\right)=\left\{\begin{array}{c}
R_{0, M}\left(E / N_{J}\right), \quad E / N_{J}<2.871 \\
1-\log _{M}\left\{1+\frac{(M-1) 1.424}{E / N_{J}}\right\} \\
E / N_{J} \geq 2.871 .
\end{array}\right.
$$

This result has the same form as the mismatched cutoff rate calculated in [9] and [7] with the factor of 1.424 replaced by $4 e^{-1}=1.4715$. The cutoff rate defined in [7] is based on a suboptimal (square-law combining) decoding rule, while the cutoff rate given in (34) is based on a maximum likelihood decoding rule. Since square-law combining is much easier to implement than log-likelihood combining, the $0.14 \mathrm{~dB}$ advantage of log-likelihood combining does not appear to merit its use in any coded system. 


$$
J_{M}(x)=\frac{1+(M-1)\left[\frac{M-2}{M-1} P_{e, s}(x)+2 \sqrt{P_{e, s}(x)\left(1-P_{e, s}(x)\right) /(M-1)}\right]}{M}
$$

where $P_{e, s}(x)$ is given by (19) with $N_{0}=0, S_{n}=1$, and $x=$ $E / N_{J}$.

\section{B. No Side Information A vailable}

With hard decisions, no side information available, and $N_{s}$ $=1$, the optimal strategy for the jammer is to maximize the average symbol error probability given in [34]. The cutoff rate is then

$$
R_{0, M}^{(1)}\left(E / N_{J}\right)=-\log _{2} J_{M}^{(1)}\left(E / N_{J}\right)
$$

where $J_{M}^{(1)}$ is given in (49) with $P_{e, s}(x)$ given in (35).

When $N_{s}>1$, the cutoff rate is given by

$$
\begin{aligned}
R_{0, M}{ }^{\left(N_{s}\right)}\left(E / N_{J}\right) \\
=\min _{0<\rho \leq 1}\left\{1-\frac{2}{N_{s}} \log _{M} \sum_{k=0}^{N_{s}}\left(\begin{array}{c}
N_{s} \\
k
\end{array}\right)(M-1)^{k}\left(\alpha_{N_{s}, k}\right)\right\}
\end{aligned}
$$

and $\alpha_{N_{s}, k}$ is given in (38).

\section{NUMERICAL RESULTS}

In this section we present numerical results to indicate the bit signal-to-noise ratio needed for reliable communication on channels with partial-band jamming. We present results for both the channel capacity and the cutoff rate.

The minimum value of $E_{b} / N_{J}$ that is needed for reliable communication is determined from the channel capacity in the following manner. The capacity is a function of the symbol signal-to-noise ratio: $C\left(E / N_{J}\right)$. From the channel coding theorem there exist codes of rate $r$ in $M$-ary units (i.e., $M$-ary information symbols/ $M$-ary channel symbols) such that reliable communication (arbitrarily small error probability) is possible, provided

$$
r<C\left(E / N_{J}\right) \text {. }
$$

This is equivalent to

$$
E / N_{J}>C^{-1}(r)
$$

The bit signal-to-noise ratio can be determined from the symbol signal-to-noise ratio by

$$
E_{b} / N_{J}=E / N_{J} /\left(r \log _{2} M\right) .
$$

Using (53) in (52) we obtain

$$
E_{b} / N_{J}>C^{-1}(r) / r \log _{2} M
$$

as the necessary condition for reliable communication. The interpretation is that there exist codes of rate $r$ such that the error probability will be arbitrarily small, provided (54) is satisfied. This obviously does not say that all codes of rate $r$ will have error probability arbitrarily small for all $E_{b} / N_{J}$ satisfying (54), only that there exist such codes. Furthermore, from the converse to the coding theorem, we know that no codes exist that have error probability arbitrarily small with rate greater than capacity. This is equivalent to saying that no code of rate $r$ can have error probability arbitrarily small with $E_{b} / N_{J}$ less than the right side of (54). Thus, (54) is a necessary condition for any code of rate $r$ to have arbitrarily small error probability. In the numerical ratio for reliable communication as a function of the code rate $r$.

If $E / N_{J} \geq \widehat{T}_{M}$, then using (28) and (54) we have

$$
r<1-\frac{\bar{\Upsilon}_{M}-\bar{\Upsilon}_{M} C_{M}\left(\bar{\Upsilon}_{M}\right)}{E / N_{J}}
$$

or

$$
E_{b} / N_{J}>\frac{\bar{\Upsilon}_{M}-\bar{\Upsilon}_{M} C_{M}\left(\bar{\Upsilon}_{M}\right)}{r(1-r)}
$$

as the necessary condition for reliable communication. If we wish to use the best possible codes, then we choose the code rate that minimizes the energy necessary for reliable communications. The minimization of (56) is easy and yields the optimum code rate of $1 / 2$. For this code rate the signal-tonoise ratio necessary for reliable communication becomes

$$
E_{b} / N_{J}>4\left[\bar{\Upsilon}_{M}-\bar{\Upsilon}_{M} C_{M}\left(\bar{\Upsilon}_{M}\right)\right] / \log _{2} M \text {. }
$$

In (55) and (56) it was assumed that $E / N_{J}>\overrightarrow{\boldsymbol{\Upsilon}}_{M}$. So, provided $C_{M}\left(\widehat{\Upsilon}_{M}\right)<1 / 2$, optimal codes have rate $1 / 2$. So far we have not specified the type of receiver (e.g., hard decision, soft decisions), but only that side information is available. For the soft decision receiver the capacity is given in (30) for $M=2$. For this case we have that $\Upsilon_{2}=2.4137$ and $\widetilde{\Upsilon}_{2}\left[1-C_{2}\left(\mathbb{\Upsilon}_{2}\right)\right]=2.4137$. Notice that in this case $\widetilde{\Upsilon}_{2}=$ 2.4137 and $C_{2}\left(\widetilde{\Upsilon}_{2}\right)=0.5136>1 / 2$ so that $(55)$ and $(56)$ are not valid. The optimal code rate can in fact be found to be 0.48 with $E_{b} / N_{J}=6.72 \mathrm{~dB}$. For hard decisions and side information available, the constants for determining the capacity in (39) are shown in Table $I$. For $M=2,4,8,16$, and $32, C_{M}\left(\Upsilon_{M}\right)$ is less than $1 / 2$. This means the best codes have rate $1 / 2$. The minimum required signal-to-noise ratio $\left(E_{b} / N_{J}\right)_{\min }$ is given as the right-hand side of (57). This is also listed in Table $I$.

When side information is unavailable and hard decisions are made, the capacity is given by (36). The values of $\lambda_{M}$, $C_{M}\left(\lambda_{M}\right)$ are given in Table II. The optimal code rate without side information can be calculated in a similar fashion to the calculation with side information (see [19]). The optimal code rate depends on $M$ and is given in Table II.

Some researchers consider the cutoff rate as the upper limit of code rates for which "practical" and reliable coding schemes exist. For channels with memory, however, it does not seem that the cutoff rate is a good measure of the code rates for which practical coding schemes exist (see [13]). If we use the cutoff rate as a measure of the channel, the same analysis yields

$$
E_{b} / N_{J}>R_{0, M}{ }^{-1}(r) / r \log _{2} M
$$

as the necessary condition for "practical" and reliable communication. As with channel capacity, there is an optimal code rate which minimizes the energy per information symbol necessary for reliable (and practical) communications. The optimal code rates can be found [19] for each value of $M$ and are given in Table III along with the minimum value of $E_{b} / N_{J}$ for soft decisions with side information. In Table IV the constants used in (46) to evaluate the cutoff rate with hard decisions and side information are given along with the minimum required signal-to-noise ratio. In Table $V$ we 
TABLE I

CONSTANTS FOR DETERMINING CAPACITY WITH HARD DECISIONS AND SIDE INFORMATION AVAILABLE AND OPTIMAL CODE RATES

\begin{tabular}{rcccc}
\hline \multicolumn{1}{c}{$\Upsilon_{M}$} & $\Upsilon_{M}$ & $C_{M}\left(\Upsilon_{M}\right)$ & $\left(E_{b} / \bar{N}_{J}\right)_{\min }(\mathrm{dB})$ & $r_{\mathrm{opt}}$ \\
\hline 2 & 3.017 & 0.4982 & 7.82 & 0.50 \\
4 & 3.275 & 0.4878 & 5.26 & 0.50 \\
8 & 3.661 & 0.4721 & 4.11 & 0.50 \\
16 & 4.015 & 0.4540 & 3.41 & 0.50 \\
32 & 4.388 & 0.4354 & 2.97 & 0.50 \\
\hline
\end{tabular}

TABLE II

CONSTANTS FOR DETERMINING CAPACITY WITH HARD DECISIONS AND NO SIDE INFORMATION AVAILABLE AND OPTIMAL CODE RATE

\begin{tabular}{rccccc}
\hline \hline$M$ & $\lambda_{M}$ & $P_{e, s}\left(\lambda_{M}\right)$ & $C_{M}\left(\lambda_{M}\right)$ & $\left(E_{b} / N_{J}\right)_{\min }(\mathrm{dB})$ & $r_{\mathrm{opt}}$ \\
\hline 2 & 2.000 & 0.184 & 0.311 & 7.98 & 0.379 \\
4 & 2.383 & 0.293 & 0.331 & 5.48 & 0.396 \\
8 & 2.782 & 0.369 & 0.338 & 4.28 & 0.410 \\
16 & 3.194 & 0.426 & 0.338 & 3.59 & 0.422 \\
32 & 3.613 & 0.472 & 0.333 & 3.17 & 0.431 \\
\hline
\end{tabular}

TABLE III

OPTIMAL CODE RATES (FROM CUTOFF RATE) AND MINIMUM SIGNALTO-NOISE RATIO WITH SOFT DECISIONS AND SIDE INFORMATION AVAILABLE

\begin{tabular}{rccc}
\hline \hline$M$ & $\left(E_{b} / N_{J}\right)_{\min }(\mathrm{dB})$ & $r_{\text {opt }}$ & $\rho_{\text {opt }}$ \\
\hline 2 & 8.33 & 0.454 & 0.927 \\
4 & 6.14 & 0.405 & 0.861 \\
8 & 5.20 & 0.355 & 0.813 \\
16 & 4.74 & 0.308 & 0.781 \\
32 & 4.52 & 0.266 & 0.763 \\
\hline
\end{tabular}

TABLE IV

OPTIMAL CODE RATES (FROM CUTOFF RATE) AND MINIMUM SIGNALTO-NOISE RATIO WITH HARD DECISIONS AND SIDE INFORMATION AVAILABLE

\begin{tabular}{rccccc}
\hline$M$ & $\sigma_{M, 1}$ & $\Sigma_{M, 1}$ & $\left(E_{b} / N_{J}\right)_{\min }(\mathrm{dB})$ & $r_{\mathrm{opt}}$ & $\rho_{\text {opt }}$ \\
\hline 2 & 4.268 & 1.007 & 9.84 & 0.454 & 0.974 \\
4 & 4.224 & 1.502 & 7.67 & 0.405 & 0.895 \\
8 & 4.245 & 1.825 & 6.86 & 0.355 & 0.822 \\
16 & 4.314 & 2.063 & 6.64 & 0.308 & 0.760 \\
32 & 4.460 & 2.291 & 6.72 & 0.266 & 0.714 \\
\hline
\end{tabular}

give the corresponding constants for the cutoff rate with hard decision and no side information when the memory length $N_{s}$ $=1$.

We will compare our results for partial-band jamming with the results for the uniform jamming case (i.e., $\rho=1$ ). In all figures we will plot the lower bound in $(60)$ or $(61)$ as a function of the code rate. In Fig. $2, E_{b} / N_{J}$ necessary for reliable communication is shown for binary FSK with soft decisions (side information available). Notice that for small code rates $(<0.513)$, the optimal strategy for the jammer is uniform jamming $(\rho=1)$. The fact that for small code rates $E_{b} / N_{J}$ increases is due to the noncoherent combining loss encountered with noncoherent reception of binary FSK. The same results for binary phase-shift keying with coherent
TABLE V

OPTIMAL CODE RATES (FROM CUTOFF RATE) AND MINIMUM SIGNALTO-NOISE RATIOS WITH HARD DECISIONS AND NO SIDE INFORMATION AVAILABLE

\begin{tabular}{rrrr}
\hline$M$ & $\left(E_{b} / N_{J}\right)_{\min }(\mathrm{dB})$ & $r_{\mathrm{opt}}$ & $\rho_{\mathrm{opt}}$ \\
\hline 2 & 10.40 & 0.247 & 0.738 \\
4 & 8.24 & 0.239 & 0.746 \\
8 & 7.43 & 0.228 & 0.734 \\
16 & 7.16 & 0.215 & 0.713 \\
32 & 7.18 & 0.205 & 0.689 \\
\hline
\end{tabular}

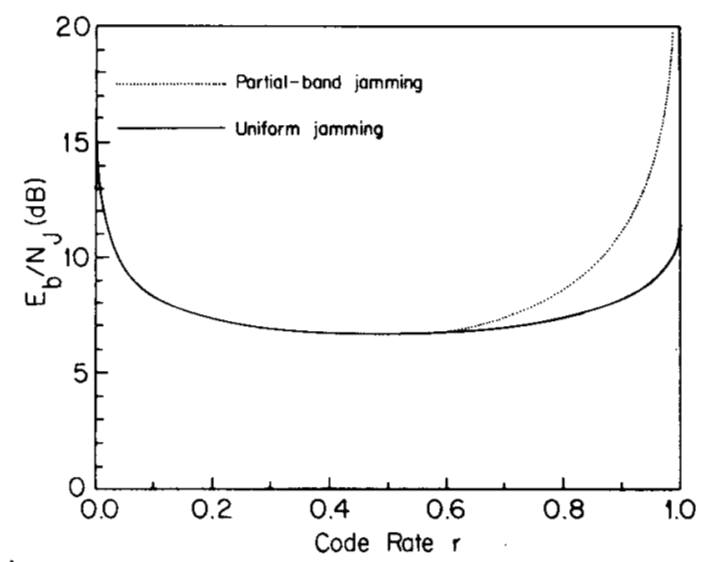

Fig. 2. $E_{b} / N_{J}$ needed to achieve capacity for binary FSK with soft decisions (side information available).

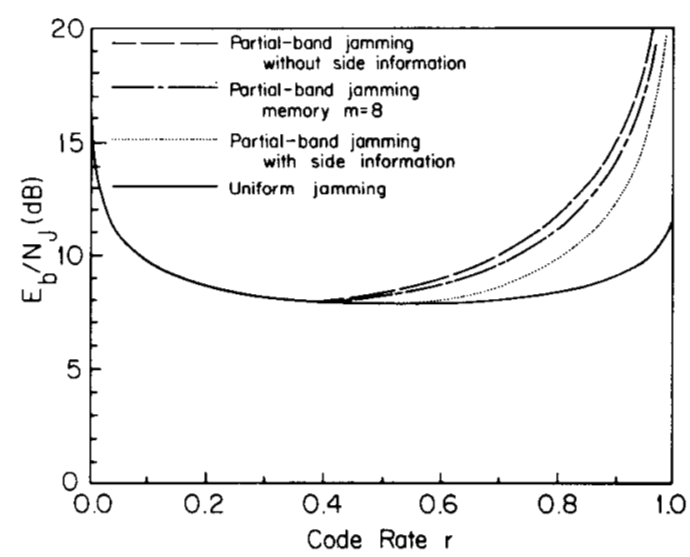

Fig. 3. $E_{b} / N_{J}$ needed to achieve capacity for binary FSK with hard decisions.

reception ([20] or [15]) show that the necessary signal-tonoise ratio decreases as the code rate decreases approaching the limit of $-1.59 \mathrm{~dB}$.

In Fig. 3 the corresponding results are shown for hard decision FSK. In this figure both cases of side information availability are shown. Notice again that for small rates uniform jamming is optimal, which means side information is not needed, but for large rates side information can be worth between 1 and $5 \mathrm{~dB}$. Also shown in Fig. 3 is the case of no side information and memory 8 ( $N_{s}=8$ bits/hop). This is slightly better than the case $N_{s}=1$. Without side information as $N_{s} \rightarrow \infty$, the $E_{b} / N_{J}$ necessary for reliable communication approaches $E_{b} / N_{J}$ necessary for reliable communication when side information is available [13]. In Figs. 4-6 we show the corresponding results for $M=8,16$, and 32 .

Numerical results for the cutoff rate parameter are given in 


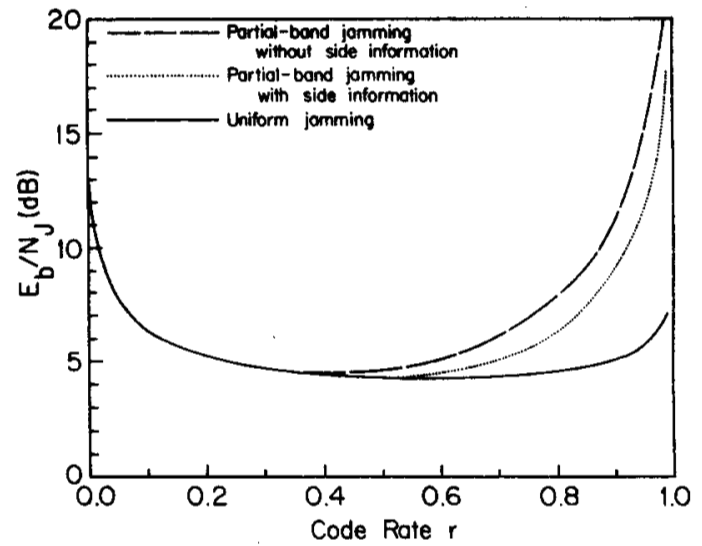

Fig. 4. $E_{b} / N_{J}$ needed to achieve capacity for 8-ary FSK with hard decisions.

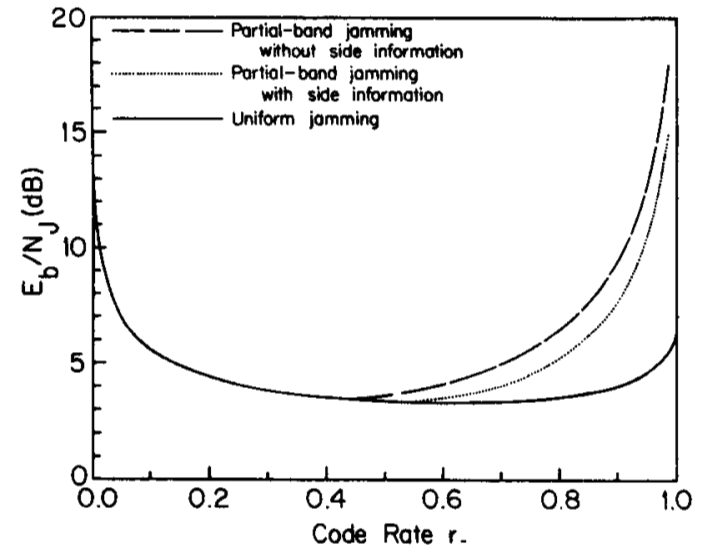

Fig. 5. $E_{b} / N_{J}$ needed to achieve capacity for 16-ary FSK with hard decisions.

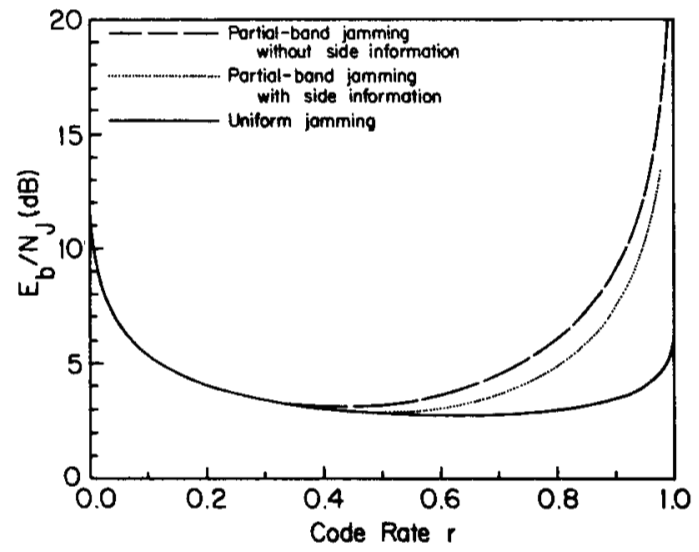

Fig. 6. $E_{b} / N_{J}$ needed to achieve capacity for 32-ary FSK with hard decisions.

Figs. 7-9. In Fig. 7 the results for binary FSK with soft decisions (side information available) is shown. In Fig. 8 the results for hard decisions are shown. In this figure we also show the case of memory 5 and 10 with and without side information. Notice that increasing $m$ decreases the cutoff rate or increases the necessary signal-to-noise ratio to achieve the cutoff rate. This is a general phenomenon of the cutoff rate [13]. That is, the larger the channel memory, the smaller the cutoff rate, while the opposite is true for channel capacity. Finally, in Fig. 9 the results for 32-ary FSK are shown.

In Part II of this paper we will determine the performance

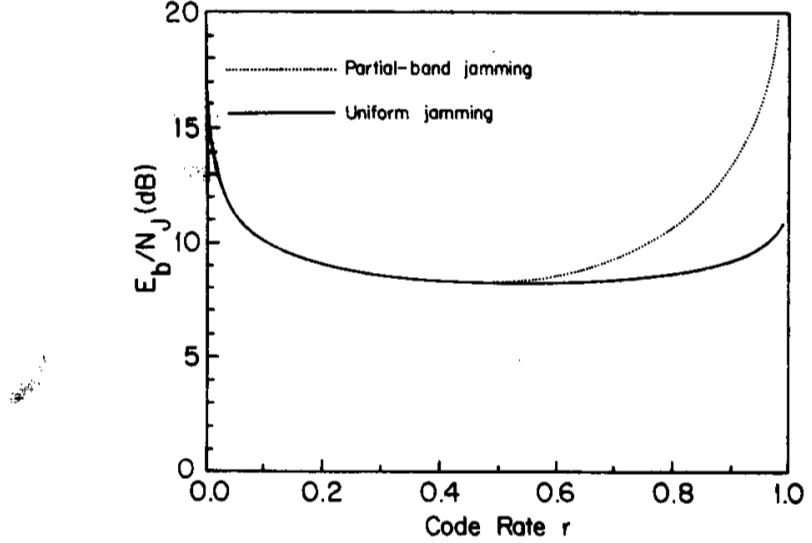

Fig. 7. $E_{b} / N_{J}$ needed to achieve cutoff rate for binary FSK with soft decisions (side information available).

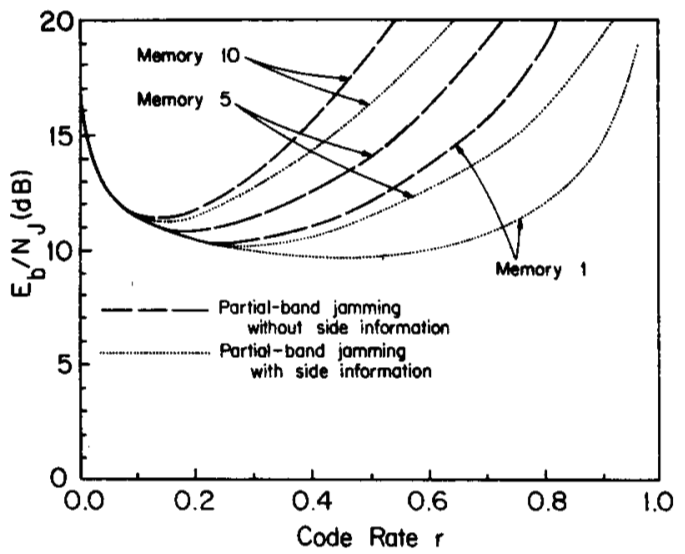

Fig. 8. $E_{b} / N_{J}$ needed to achieve cutoff rate for binary FSK with hard decisions.

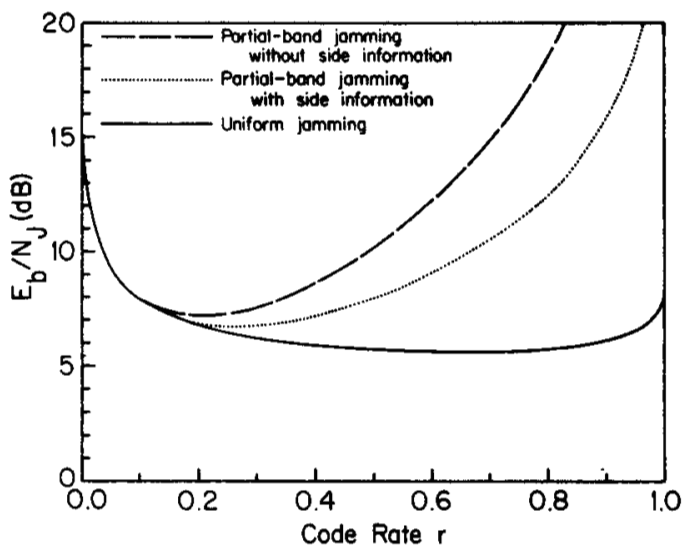

Fig. 9. $E_{b} / N_{J}$ needed to achieve cutoff rate for 32-ary FSK with hard decisions.

of several coding schemes for these channels. We will also compare capacity and cutoff rate results to the actual performance of codes.

\section{APPENDIX}

In this Appendix we give a justification of $C=\max \min$ $I\left(X^{(n)} ; Y^{(n)}, S_{n}\right)$ and $\bar{C}=\max \min I\left(X^{(n)} ; Y^{(n)}\right)$ as channel capacity with and without side information. We assume that the jammer's strategy is such that, after determining his optimal fraction of the frequency band to jam, the fraction is fixed. That is, $\rho$ is fixed for an entire codeword. In this way the channel is easily seen to be a compound channel. In [21] 
it is shown that for finite input and output compound channels with input $X$ and output $Y$, there exist codes with rate $r$ for which the error probability goes to zero as the block length goes to infinity, provided $r<\max \min I(X ; Y)$. For infinite output alphabet the results are still true, provided the receiver knows which channel the jammer has selected [22]. If this is not the case, then the results are upper bounds on the channel capacity and lower bounds on the minimum $E_{b} / N_{J}$ necessary for reliable communication.

Another possible strategy for the jammer is to select a new channel (value of $\rho$ ) for each symbol of a codeword. With this strategy the channel becomes an arbitrarily varying channel (AVC). The capacity of a general AVC is not known. However, for a binary input, binary output channel there exist codes with rate less then $\max \min I(X ; Y)$ for which the (maximum) error probability of any codeword goes to zero as the block length goes to infinity [23]. In this case the compound channel and the AVC have the same capacity. For nonbinary AVC's the capacity is not known.

\section{REFERENCES}

[1] S. W. Houston, "Modulation techniques for communication-Part 1 Tone and noise jamming performance of spread spectrum $M$-ary FSK and 2, 4-ary DPSK waveforms," in Proc. IEEE Nat. Aerosp. Electron. Conf., June 1975, pp. 51-58.

[2] B. K. Levitt and J. K. Omura, "Coding tradeoffs for improved performance of FH/MFSK systems in partial band noise," in Nat. Telecommun. Conf. Rec., Dec. 1981, pp. D9.1.1-D9.1.5.

[3] W. E. Stark and R. J. McEliece, "Capacity and coding in the presence of fading and jamming," in Nat. Telecommun. Conf. Rec., Nov. 1981 , pp. B7.4.1-B7.4.5.

[4] A. J. Viterbi and I. M. Jacobs, "Advances in coding and modulation for noncoherent channels affected by fading partial band, and multipleaccess interference," in Advances in Communication Systems, vol. 4. New York: Academic, 1975, pp. 279-308.

[5] E. A. Bucher, "Coding options for efficient communication on nonstationary channels," in Nat. Telecommun. Conf. Rec., Dec. 1980, pp. $4.1 \cdot 1-4.1 .7$

[6] J. B. Cain and J. M. Geist, "Modulation, coding and interleaving tradeoffs for spread spectrum systems," in Nat. Telecommun. Conf. Rec., Nov. 1981, pp. B7.3.1-B7.3.5.

[7] J. K. Omura and B. K. Levitt, "A general analysis of anti-jam communication systems," in Nat. Telecommun. Conf. Rec., Dec. 1981, pp. B7.1.1-B7.1.5.

[8] S. L. Bernstein, H. M. Heggestad, S. Mui, and I. Richer, "Variable rate Viterbi decoding in the presence of RFI," in Nat. Telecommun. Conf. Rec., Dec. 1977, pp. 36.6.1-36.6.5.

[9] A. J. Viterbi, "Spread-spectrum communications-Myths and realities," IEEE Commun. Mag., vol. 4, pp. 11-18, May 1979.

[10] G. C. Clark and J. B. Cain, Error-Correction Coding for Digital Communications. New York: Plenum, 1981.

[11] W. E. Stark, "Coding for frequency-hopped spread-spectrum communication with partial-band interference-Part II: Coded performnance," this issue, pp. 1045-1057.

[12] E. A. Geraniotis and M. B. Pursley, "Error probabilities for slowfrequency-hopped spread-spectrum multiple access communications over fading channels," IEEE Trans. Commun., vol. COM-31, pp. 996-1009, May 1982.

[13] R. J. McEliece and W. E. Stark, "Channels with block interference," IEEE Trans. Inform. Theory, vol. IT-30, pp. 44-53, Jan. 1984.

[14] J. K. Omura and B. K. Levitt, "Coded error probability evaluation for antijam communication systems," IEEE Trans. Commun., vol. COM-30, pp. 896-903, May 1982

[15] W. E. Stark, "Coding for frequency-hopped spread-spectrum channels with partial-band interference," Ph.D. dissertation, Univ. Illinois, Urbana, Coord. Sci. Lab. Rep. R-945, July 1982.

[16] R. J. McEliece, The Theory of Information and Coding. Reading, MA: Addison-Wesley, 1977.

[17] S. A. Butman, I. Bar-David, B. K. Levitt, R. F. Lyon, and M. J. Klass, "Design criteria for noncoherent Gaussian channels with MFSK signaling and coding," IEEE Trans. Commun., vol. COM-24, pp. 1078-1088, Oct. 1976.

[18] K. L. Jordan, "The performance of sequential decoding in conjunction with efficient modulation," IEEE Trans. Commun. Technol., vol. COM-14, pp. 283-297, June 1965.

[19] R. J. McEliece and W. E. Stark, "The optimal code rate vs. a partial band jammer," in Proc. IEEE Military Commun. Conf., Oct. 1820, 1982, vol. 1, pp. 8.5.1-8.5.4.

[20] - "An information theoretic study of communication in the presence of jamming," in Rec. IEEE Int. Conf. Commun., June 1981, pp. 45.3.1-45.3.5.

[21] J. Wolfowitz, Coding Theorems of Information Theory, 3rd ed. Berlin, Germany: Springer-Verlag, 1978.

[22] H. Kesten, "Some remarks on the capacity of compound channels in the semicontinuous case,"'Inform. Contr., vol. 4, pp. 169-184, 1961.

[23] I. Csiszar and J. Korner, Information Theory: Coding Theorems for Discrete Memoryless Systems. New York: Academic, 1981.

Wayne E. Stark (S'77-M'78), for a photograph and biography, see p. 774 of the August 1985 issue of this TRANSACTIONS. 